\title{
An Opportunity to Share
}

\section{Maria C. Bishop ${ }^{1}$}

Published online: 26 July 2019

(C) American Association for Cancer Education 2019

I often leave cancer education conferences very impressed by the quality of mentorship and inspired by our colleagues' work around the world. I am also reminded that the work presented represents only a small sample of what our global cancer education community contributes to decreasing the burden of cancer for our patients, families, and communities. While attending the 2019 European Association for Cancer Education (EACE) annual scientific meeting in Porto, Portugal, I had the opportunity to meet with researchers, healthcare providers, and students to learn more about their work. I would like to highlight the educational programs that were shared with me and are available to you and your students. The Journal of Cancer Education (JCE) has a column that features announcements of educational programs, fellowships, and grants. With contributions from researchers, I hope to bring attention to this column by highlighting existing programs and encouraging others to contribute.

Over the past year, we received manuscripts from nearly every continent, with topics ranging from cross-cultural medical care education to the impact of genetic counseling educational tools. I believe this reflects a global commitment to cancer education. Many of the manuscripts and published abstracts were the result of work funded by the National Cancer Institute (NCI). Three examples of NCI-funded cancer education programs are the opportunities offered by American Association for Cancer Education (AACE) members Dr. Suzanne Gronemeyer, Dr. Cathy Meade, and Dr. Amr Soliman.

Since 1997, Dr. Gronemeyer has been the Program Director and Principal Investigator on the R25-funded Pediatric Oncology Education (POE) program at St. Jude Children's Research Hospital. The POE program offers a unique opportunity for students preparing for careers in the

Maria C. Bishop

mbishop@email.arizona.edu

1 Division of Hematology and Oncology, University of Arizona Cancer Center, 1515 N Campbell Ave, Tucson, AZ 85724, USA biomedical sciences, medicine, nursing, pharmacy, psychology, or public health to gain biomedical and oncology research experience. The POE program provides a short-term training experience (internship) in either laboratory or clinical research. A primary goal of the POE program is to encourage students to pursue a career in cancer research. Since its founding in 1978, 86\% of program alumni have obtained doctorates. Members of underrepresented groups and women are particularly encouraged to apply, since another long-term goal of their program is to increase the diversity of persons engaged in oncology research and practice.

Dr. Meade is a collaborator on the Enriching Communication Skills for Health Professionals in Oncofertility (ECHO) R25 program. The goal of the project is to develop a distance learning-based educational program for nurses and allied health professionals in oncology care settings. As survival improves among adolescents and young adults with cancer, there is a greater need to address qualityof-life issues, particularly in the area of reproductive health. The NCI-funded ECHO project is a web-based training program focusing on building communication skills. Training topics include the risk of infertility, fertility preservation, sexual functioning, body image, contraception, and ethical, social, and cultural considerations in reproductive health. Eligible participants include nurses, physician assistants, psychologists, and social workers who work in oncology settings and care for at least five cancer patients (ages 15-45) per year. (MPIs: Susan Vadaparampil/Gwendolyn Quinn - R25 CA142519).

Dr. Soliman is the Principal Investigator, and Dr. Robert Chamberlain serves as the Coinvestigator, on the R25-funded Cancer Epidemiology Education in Special Populations (CEESP) program. The program provides funding for $15 \mathrm{stu}-$ dents to travel and conduct summer research in US minority and global settings. It also provides mentored research training focused on cancer epidemiology and cancer prevention. The program is open to graduate students (MPH, MSc, $\mathrm{PhD}$, and DrPH candidates) from all schools and programs of public health in the USA. Previous internships have been conducted 
in Ghana, Tanzania, Uganda, Morocco, Vietnam, Kenya, Egypt, Tunisia, Zambia, Cyprus, Peru, Mexico, and the USA. Students have conducted research in a variety of topics, including breast and cervical cancers. Over $150 \mathrm{MPH}$ and $\mathrm{PhD}$ students have been trained since 2006, and over $60 \%$ of the alumni of the program have pursued studies in cancer epidemiology and cancer prevention and control in $\mathrm{PhD}$ and postdoctoral programs.

Our EACE colleagues, Dr. Jakob de Vries, Dr. Sabine Fromm-Haidenberger, and Dr. Schelto Kruijff, contributed to the development of a longstanding summer school program. The first International Summer School Oncology for Medical Students (ISOMS) was held in 1996 at University Medical Center Groningen (UMCG) in the Netherlands. For two decades, ISOMS has collaborated on a biannual summer program alternating with the Vienna Summer School on Oncology (VSSO) at the Medical University of Vienna. The programs are designed to increase the knowledge of medical students from around the world in general cancer care, to reduce their fear of cancer, and to create exposure to cancer-related problems. Course aims include introducing cancer research and development, new technologies in diagnostic procedures, and modern multidisciplinary treatment approaches. The course also provides opportunities for students to become familiar with preparing abstracts and posters and presenting at international meetings. The program is designed to encourage critical scientific reasoning and the development of collaboration and communication skills with peers from all around the world.

Another AACE member, Dr. Shine Chang at The University of Texas MD Anderson Cancer Center in Houston, is the contact Principal Investigator on a postdoctoral program in cancer prevention that is available to US and international students. The program's emphasis is on cancer prevention research training and education, integrating mentorship, trainee diversity, career development, and interdisciplinary collaboration. Recruitment of women and minority trainees has been highly successful. Their program continues to maintain a unique emphasis on supporting trainees who are of the first generation in their family to attend college. Their program also leverages MD Anderson's Extension for Community Healthcare Outcomes (ECHO) program to connect their trainees with cancer prevention professionals and practitioners around the world and in Texas, especially in underserved regions such as the Rio Grande Valley. Trainees can gain valuable insights into research and application of cancer prevention, as well as career possibilities in a variety of settings, while forging collaborative relationships with other professionals in these remote sites.

The new AACE-funded Research, Education, Advocacy, and Direct Service (READS) program provides small grants to active AACE members in good standing who champion the highest standards for global cancer education through evidence-based practices to achieve quality outcomes. The grants are intended to assist members in becoming more involved in research, education, teaching, and advocacy as well as direct service on national and international levels. It was established in 2019 to support AACE members in their cancer education goals.

This is just a small sample of our colleagues' inspiring work. These programs, fellowships, and grants provide opportunities to participate in their educational offerings, and the authors are an excellent resource for projects you are considering.

Our next editorial will be written by the EACE Presidentelect, Dr. Radoslaw Tarkowski. Dr. Tarkowski is an educator, mentor, and surgical oncologist. His expertise is in breast cancer surgery, and he recently wrote a book that serves as a teaching guide for surgeons to learn new breast surgery techniques. I think you will enjoy reading about cancer education from a surgeon's perspective.

The 2019 International Cancer Education Conference (ICEC) will be held in Salt Lake City, Utah, on September 18-20. The theme of the conference is "Revolutionizing Cancer Education Globally Through Technology Across Generations." Among many other activities, we will have a mentoring session; please refer to the website and registration form for additional details. The conference website (2019.attendicec.org) also has a link to available scholarships and grants. Looking ahead, the EACE is hosting their next annual scientific meeting in Krakow, Poland on April 1-3, 2020. I look forward to seeing many of you at both events. Your contributions to the JCE column that features announcements of educational programs, fellowships, and grants are always encouraged!

Publisher's Note Springer Nature remains neutral with regard to jurisdictional claims in published maps and institutional affiliations. 